\title{
WHAT HAPPENS WHEN DESIGNERS DON'T PLAY BY THE RULES: TOWARDS A MODEL OF OPPORTUNISTIC BEHA VIOUR IN DESIGN
}

\author{
A. Khushalani ${ }^{1}$, R. Smith ${ }^{1,2}$ and S. Howard ${ }^{1}$ \\ ${ }^{1}$ Centre for Systems Methodologies, Department of Computer Science, \\ Swinburne University of Technology, Hawthom, Victoria 3122, Australia; \\ ${ }^{2}$ Department of Information Systems, Monash University, \\ Caulfield, Victoria 3145, Australia.
}

\begin{abstract}
It is being recognised increasingly in the scientific literature that real world design problem solving is non hierarchical, being characterised by a strategy, termed 'opportumism', whereby designers discover and/or adapt their problem solving goals and activities, in response to the state of the problem and the environment in which that problem exists. This paper explores the concept of opportunism more formally than has hitherto been attempted. We adopt an approach (which we term "TheoryBuilder") which is a hybrid of the theory testing and theory building approaches. The TheoryBuilder appraach taken adapts the Lancaster Soft Systems Methodology to generate an initial model of opportunistic bebaviour, to be refined by subsequent iterative comparison with the existing literature and empirical studies. The utility of the initial model is demonstrated by constructing a list of the major cognitive elements for all activities appearing in this initial model, and then using it to analyse a sample verbal protocol collected from an empirical study observed by the authors. Initial results are very promising, supporting the need for more comprehensive examination and refinement of the model. Possible future research directions are discussed.
\end{abstract}

\section{INTRODUCTION}

Whilst impressive developments in computer support tools (eg, CASE and CAD) have enabled designers in various fields to generate final products of apparently improved quality, it may be significant that only limited contributions have been made to the provision of support to designers undertaking what might be considered the more innovative, up-stream design tasks (eg, problem decomposition and solution searching). One interpretation might attribute this to the relatively impoverished state of our present understanding of the strategies designers employ when they seek to understand and solve design problems.

Research in recent years into design problem solving strengthens the conviction that the use of many design methodologies is 'opportunistic', being characterised by frequent discovery and/or adaptation of goals and activities, in response to changing circumstances. Such is hardly surprising given the obstacles which exist to rigorous application of top-down design problem solving methodologies (eg, incomplete and vague requirements, real world complexity, the inability of designers to comprehend and track all details, and the preconceived ideas of designers (Pamas \& Clements, 1986)). The current literature, however, provides a confused picture of opportunism.

In the present paper, we seek to address this shortcoming through development of a model of opportunistic behaviour. Against a background discussion of the notion of design problem solving, and a substantial review of the present literature on opportunistic behaviour, we synthesise a definition of opportunism, which we use subsequently to stimulate development of an initial model of opportunistic bebaviour, generated using an adapted form of the Lancaster Soft System Methodology. Some initial attempts to use the model to structure the analysis of sample verbal protocols collected from design studies observed by the authors, are reported. Finally, we speculate on the ongoing research process required to iteratively refine the model.

\section{DESIGN PROBLEM SOLVING}

In the context of this paper, design is to be viewed as the process of problem understanding and problem solving with the aim of producing an artefact. To establish the context in which we study opportunistic behaviour we first examine the present literature which addresses, in turn, the notion of a design problem, the processes undertaken by design problem solvers, and the strategies they adopt. 


\section{Design Problems}

Problems have been conveniently classified on a continuum between the extremes of 'hard' and 'soft' (Checkland, 1981). A 'hard' problem is one which can be formulated as a search for an efficient means of achieving a defined end (eg, how should an engineer construct a bridge to convey specified vehicles across a specified gorge?), whereas a 'soft' problem is one in which ends, goals and purposes are themselves problematic (eg, how should the Australian Government address the problem of unemployed youth on our streets?), and so it is not meaningful to talk about means-ends relationships.

This terminology has not generally been used in the design literature, where instead problems are characterised as either well-structured (equivalent to 'hard'), or ill-structured (essentially equivalent to 'soft'). Simon (1973) has suggested however that 'the boundary between well-structured and illstructured problems is vague, fluid and not susceptible to formalisation', consistent with Checkland's notion of a continuum.

A considerable literature now suggests that real-world design problems (eg, organisational design, mechanical engineering design, software design) are difficult to express comprehensively, as many components of the problem will not be visible until some attempt is made at generating solutions (Lawson, 1980). Further, it is observed (Bisseret et al., 1988; Carroll et al., 1979; Davies, 1989; Guindon, 1990a; Malhotra et al., 1980; Visser, 1991b) that: generation of solutions will lead to emerging implications, causing objectives and priorities to change; designers perceive problems differently and are likely to formulate different solutions to the same design problem; in principle there is an infinite number of solutions to the same design problem; and optimal solutions do not necessarily exist. In short, design problems in the real world generally lie towards the ill-structured or 'soft' end of the continuum. It seems clear then, that any complete and systematic understanding of design problem solving will need to account for the effects of different problem classes. Furthermore, any understanding that can be applied to the real world will need to be grounded in ill-structured domains.

\section{The Design Problem Solving Process}

Numerous studies present a variety of views of the design problem solving process. Lawson (1980) characterises it as never-ending in principle, as a consequence of the observation that typically design problems defy comprehensive description and possess numerous possible solutions. The end of the process is solely a judgement of the designer. Alternative views have it that design problem solving is a series of cycles (Carroll et al., 1979), iterative stages (Malhotra, 1980) or plans built upon a kernel idea (ie., designers pursue a single conceptual design very early and adapt it accordingly to accomplish a solution state (Guindon, 1990b; Kant, 1985; Kant \& Newell, 1984; Ullman et al., 1988)).

All agree that design problem solving deals, with different emphases, with the following set of issues: problem identification (in the context of a pre-existing unsatisfactory situation); problem definition; problem exploration; analysis (resolving the problem into simpler elements); generation of alternative solutions; evaluation of alternatives; selection of a solution; sequencing of tasks to achieve the selected solution; implementation of the solution; and assessment of whether the chosen solution has corrected/improved the identified problem, which has in turn addressed the pre-existing unsatisfactory situation (Eunson, 1988; Huber, 1980; Visser, 1990c).

In summary, the design problem solving process, whether viewed as cycles, stages or plans built around a kemel idea, is dynamic and evolutionary, requiring continuous adaptation by designers to deal with a broad spectrum of issues.

\section{Design Strategies}

A major topic of present debate concerns both the strategy (ie., the blueprint for structuring the taking of decisions by designers during design problem solving, whether it be top-down, bottom-up, depthfirst, breadth-first, simulation, analogy, reuse, ...) employed by designers during the design problem solving process, and how the choice of strategies influences solution development.

Several design studies (Adelson \& Soloway, 1985; Davies, 1991; Jeffries et al., 1981; Kant \& Newell, 1984) support the existence of a top-down strategy whereby design problems are progressively decomposed into smaller, manageable sub-problems, however this research was typically conducted under restricted conditions, generally in the software development domain involving novice programmers working on simplified problems, thereby raising questions as to whether the strategies identified could legitimately be mapped to those used by experts working on real-world problems (Visser, 1991b; Visser \& Hoc, 1990a). 
A number of studies conducted in a variety of domains over the past few years have questioned such a top-down view of the design problem solving strategy. It is beyond the scope of the present paper to report a complete review of this rapidly growing literature, however, the interested reader might consult: eg, Guindon, 1990a, 1990b; Hayes-Roth, 1980; Hayes-Roth \& Hayes-Roth, 1978, 1979; Nii, 1986; Sumiga, 1992; Visser, 1990c, 1992a, 1992b. As a result of careful consideration of this literature by the present authors, it is concluded that whilst it does not present a single, coherent definition of the behaviours being observed (generally termed 'opportunism'), the literature tends to characterise designers as employing strategies whereby they might discover new, and/or adapt their previously intended problem solving goals and activities, in response to the state of the problem and the environment in which that problem exists, at a particular point in time (ie., implying event-driven reasoning (Sumiga, 1992)).

\section{A Rationale for the Present Research}

The current research has evolved out of concern at the current conflict in the literature regarding design strategy, coupled with a perceived need to give a more formal treatment of opportunism. If indeed opportunistic behaviour is an essential component of the strategies practiced by real world designers, benefits which should accrue from such research include:

- $\quad$ provision of a framework against which to analyse some of the possibly ill-founded assumptions routinely expressed concerning design problem solving;

- development of a model which reflects the actual process, as opposed to a model which reflects a desired process; and

- identification of possible problems with current methodological approaches.

A similar sentiment has been expressed in the research agenda proposed by Broadbent (1993).

\section{A METHODOLOGY FOR GENERATING A MODEL OF OPPORTUNISM}

\section{Methodological Background}

In modelling problem solving behaviour there are two related approaches that might conceivably be pursued (Smith et al., 1994):

Theory Testing: For example, an extensive literature review could be conducted and a comprehensive model could be pieced together to represent the totality of the behaviour being reported. Hypotheses could be generated and tested through experimentation in order to refine and develop the model (see for example the work of Norcio, 1982); or

Theory Building: For example, empirical studies of the behaviour of real-world design problem solvers could be conducted and a model developed, over time, to represent the behaviour being observed (see for example the work of Shneiderman, 1986).

As discussed earlier, the current literature on opportunism provides a confused picture, mitigating against a theory testing approach. On the other hand, empirical studies provide very rich behavioural data but can be very difficult to conduct in unexplored areas, requiring a rudimentary model to guide observation. Thus theory building would be difficult without an appropriate framework.

In the present research we adopt an approach (which we term "TheoryBuilder") which is a hybrid of the two, in that it circumvents the above problems by using an adapted form of the Lancaster Soft Systems Methodology (SSM) (Checkland, 1981; Checkland \& Scholes, 1990) to propose an initial framework which would support both a coherent examination of the literature, and which will simultaneously provide the rudimentary model required to guide observation. A substantial discussion of this approach is available in Smith et al. (1994).

The TheoryBuilder approach is particularly suited to our purposes because it supports the development of systems-based models which can be usefully compared with the unstructured 'messes' which are to be found in the real-world (the present state of knowledge of opportunism could be considered one such unstructured mess). Further, it uses the language of the human activity system, the notion of purposeful human activity (ie., deliberate, decided, willed action by an individual or group) to build models (one might postulate that opportunistic behaviour can be viewed as a form of purposeful human activity), and uses the models so developed to provide a language which will support coherent debate.

TheoryBuilder should be viewed as a variant of SSM. We find it convenient to introduce this term which distinguishes both the context (ie., behavioural modelling based upon verbal protocol analysis) and additions to SSM technique (ie., use of restricted verb and noun set in the building of cognitive 
conceptual models (see Stage 3 below), and the introduction of specialised comparison techniques (see Stage 4 below)).

\section{Approach}

It is beyond the scope of the present paper to describe in any depth the procedures and techniques of the TheoryBuilder approach. Readers are referred to the paper by Smith et al. (1994), which adapts ideas and modelling techniques from SSM (Checkland, 1981; Checkland \& Scholes, 1990; Davies \& Ledington, 1991; Wilson, 1990), for a comprehensive description. In terms of the five stage representation of TheoryBuilder (Smith et al., 1994), the present study can be viewed as follows:

Stage 1 (Find out): Finding out involves displaying the perceived problematic situation such that a range of possible and hopefully relevant cognitive activity systems can be revealed. A cognitive activity system is similar to the human activity system described by Checkland (1980), but describes purposeful human activity at a cognitive level (ie., in terms of thought and consequent behaviour). In the present case, an extensive investigation of existing literature (see the discussion of design strategy above) has been conducted to establish a base of observational material on opportunism in a variety of design domains.

Stage 2 (Specify Relevant Systems): At this point we considered a range of possible input-to-outputtransformations relevant to an understanding of opportunism (eg, need to change goals and/or activities in response to events $=>$ changed goals and/or activities which represent a response to events), the possible perspectives which express the broader purpose of the transformation (eg, such changes can be deemed desirable to both process and product by designers), and environmental factors (eg, the study constraints; the existing problem situation; an established knowledge base spanning both the intended design problem solving goals/activities and the designers' wider collective knowledge and experience). In the present case, one such notional system which seemed relevant to producing insight was selected, and a root definition (a concise, tightly constructed description which makes explicit the input to output transformation, perspective taken, and environment) of the relevant cognitive activity system has been proposed.

Stage 3 (Build Cognitive Conceptual Models): A "cognitive conceptual model" is a systemic account of a cognitive activity system built on the basis of that system's root definition. It takes the form of a structured set of verbs in the imperative mode, these verbs representing the minimum necessary cognitive activities for the system to be the one named in the root definition. Thus, a cognitive conceptual model is a sub-set of the SSM type conceptual model, in that the construction of cognitive conceptual models is constrained by restricted use of verbs (eg, assimilate, understand, ...) and nouns (eg, trigger, variation, criteria, ...) related to the cognitive activity of designers. On the other hand, SSM type conceptual models can be constructed using an unrestricted set of verbs and nouns. In summary, a cognitive conceptual model is to be viewed as a model of the acquisition and manipulation of knowledge. It is important to note that the model so created does not purport to describe the real world; only to represent activities which must exist for the system to be that named in the root definition. The verbs are ordered and displayed with linking arrows according to logical contingency (by which is meant that a given activity can only commence if activities at the tails of all entering arrows have been completed). This modelling from root definition to cognitive conceptual model is the closest thing to a deterministic technique in the TheoryBuilder approach, and adopts the methods discussed extensively by authors such as Checkland \& Scholes (1990) and Davies \& Ledington (1991). In the present case, the root definition relevant to an understanding of opportunistic behaviour was mapped to a cognitive conceptual model.

Stage 4 (Compare): The cognitive conceptual model activities have then been overlaid with the real world, as previously established at Stage 1 above, complemented by our own observational studies of design problem solving. As discussed by Smith et al. (1994), a technique found useful is to create a list of cognitive elements (terms to which meaning must bave been ascribed by any actors undertaking the activities in the cognitive conceptual model for that activity to take place). For example, if a cognitive conceptual model has an activity concerned with "identifying and evaluating a possibility to vary from intended actions in response to events which act as triggers", one might identify as cognitive elements: "identified trigger", "evaluated trigger", "possibility of variation", ... (see Smith et al. (1994) for further discussion). Having identified the cognitive elements for a given cognitive conceptual model, one can probe both the existing literature, and one's own observational studies of design problem solving, for reference to such categories. The existence of references to such cognitive elements is prima facie evidence of the existence of the associated cognitive activity. 
Tabular and graphical methods designed to display the outcome of such a comparison of the cognitive conceptual model with both existing literature, and with verbal protocols collected during observational studies, have been reported by Smith et al. (1994).

In the present study, we have established a list of cognitive elements for the cognitive conceptual model developed to understand opportunistic behaviour, and we have adopted a tabular format (see Table 2 later) to display the comparison of observed behaviours with the model. Such comparison has been pursued on two bases: Can evidence be found in the literature for model activities? and, Can empirical evidence, drawn from our own observational studies, be found for model activities? Also noted, was evidence of real world activities which could not be accommodated in the model.

Stage 5 (Refine the Model): In order to establish a comprehensive and fully validated model of opportunistic behaviour, there is a need to develop the initial model to accommodate the totality of the literature and empirical material. This can be conceptualised as shown in Figure 1, as an iterative process of comparison and modification of the model to generate a closer match of the refined model to the real world.

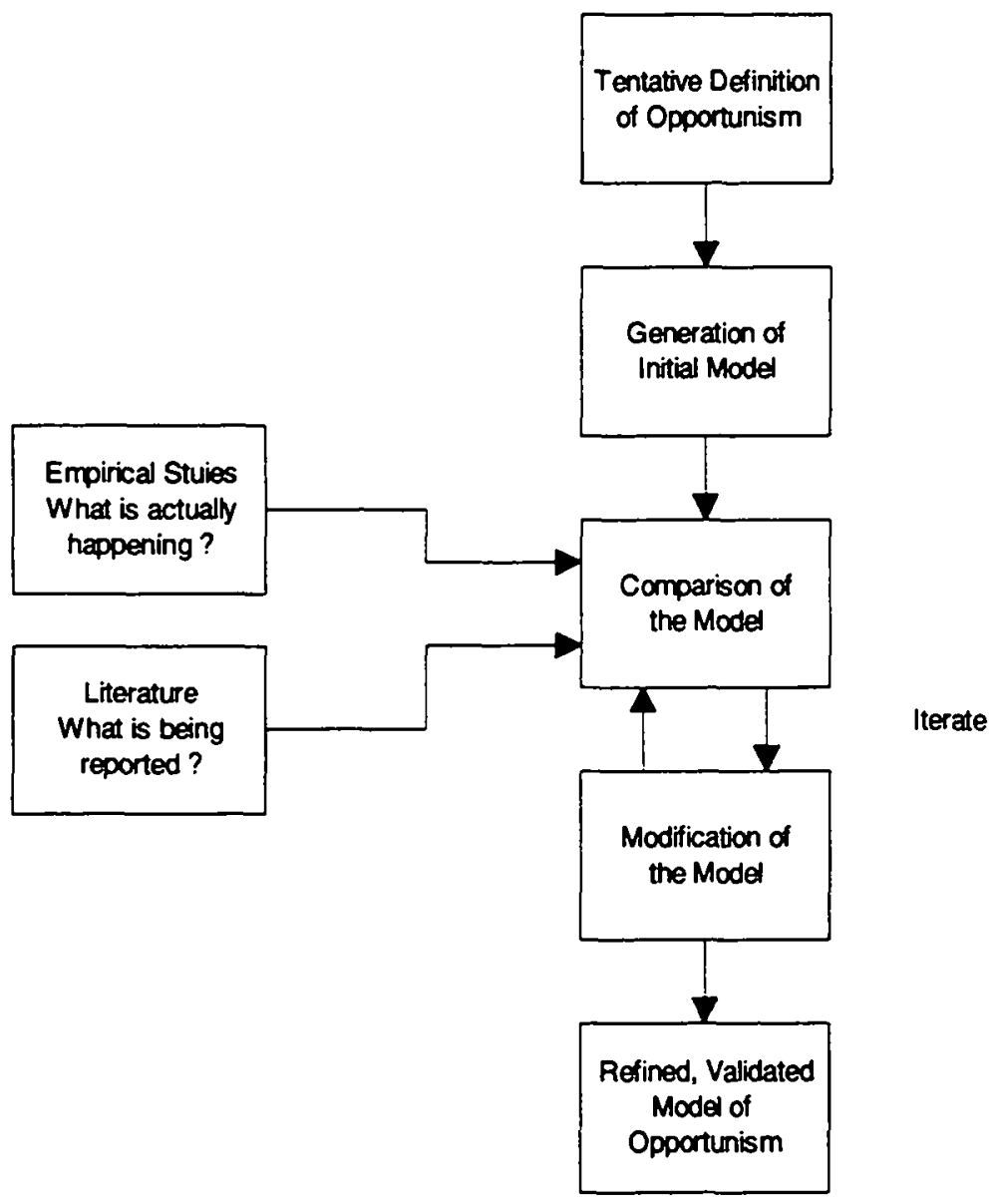

Figure 1. Model development process

\section{Naming of a Relevant System}

\section{MODEL OF OPPORTUNISM}

The making of opportunistic variations to intended design problem solving goals and activities can be viewed as a cognitive activity system, and using the root definition-cognitive conceptual model mechanisms outlined above, its minimum necessary activities can be identified. The following root definition, structured in the style recommended by Smith et al. (1994), based upon that of Checkland (1981), provides the foundation for modelling: 
A system to make opportunistic variations to intended design problem solving goals and activities: A designers' owned and operated system to make opportunistic ${ }^{*}$ variations to intended design problem solving goals and activities such that these variations could be deemed desirable both to process and product by the designers, in an environment characterised by the constraints of the study itself, the problem situation, and an established knowledge base spanning both the intended design problem solving goals and activities, and designers' wider collective knowledge and experience.

* Opportunism is to be interpreted, in accord with the discussion of the existing literature above, as: a strategy whereby design problem solvers discover and/or adapt their problem solving goals and activities, in response to the state of the problem and the environment in which that problem exists (ie., implying event-driven reasoning). It should be noted that the discovery and/or adaptation by designers often results in a changed ordering of activities, omission of activities and/or inclusion of new activities in the previously intended path.

\section{Human Activity System Based Model of Opportunism}

The minimum necessary activities demanded by the above root definition are shown in the cognitive conceptual model of Figure 2.

This model suggests that designers, in response to an identified possibility to vary from intended design problem solving goals and activities, will identify a set of possible variations, define the criteria for determining the desirability of the identified variations, evaluate according to those criteria and finally proceed to interface the variations deemed desirable and apply them.

Of the fifteen activities illustrated, activities 5 to 9 are amenable to relatively straightforward expansion to a higher level of detail, through a process of further root definition-cognitive conceptual model mapping. These elaborations of the model are displayed in Figures 3 to 7 . As a means of further clarifying the intent of each activity, we present, in Appendix A, an interpretation in terms of this model of a scenario involving opportunistic behaviour presented by Birnbaum (1985).

The cognitive conceptual model of Figure 3 suggests that designers, on perceiving a trigger or triggers, evaluate those triggers based on certain factors drawn from their individual or collective knowledge and experience (eg, a difficulty/simplicity perception strategy) thereby recognising the possibility for a variation(s) suggested by the perceived trigger(s). The term 'trigger' is here taken to mean an essential act, word, or symbol which unlocks a pattern in the receiver, thereby resulting in a flow of ideas.

The cognitive conceptual model of Figure 4 suggests that after designers have identified the possibility for variation(s) they attempt to articulate the possibility as appropriate variation(s). The notion of possible variation space introduced here is taken to mean the set of all the variations of which the designers could conceive, and its use here is intended to convey an interest by designers in exploring variation options rather than taking a single variation as granted.

The cognitive conceptual model presented in Figure 5 proposes that designers define criteria for determining the desirability of variations through a synthesis of criteria which reflect the ease with which one can conceptualise the variations, implement the variations, and predict the product benefits to be gained through application of the variations.

The cognitive conceptual model presented in Figure 6 proposes that designers, based on the desirability criteria developed in Figure 5, determine a list of variations deemed desirable. To apply those criteria, the designers must conceptualise the variations, determine the processes involved in implementing the variations, and determine the effect on product outcome of the variations. Those variations deemed desirable are then time ordered (based upon issues such as priority and contingency) and submitted to be actioned.

Finally, the cognitive conceptual model in Figure 7 suggests that designers, after determining the extent to which variations affect the previously intended design problem solving goals and activities, interface those variations to the previously intended goals and activities if necessary. Variations are then applied, together with those aspects of the previously intended design problem solving goals and activities which survive.

We emphasise again that the model in Figures 2 to 7 is an ideal type creation which expresses, in accord with the tenets of TheoryBuilder and SSM, those activities which must exist for the system to achieve the purpose expressed in the root definition. The model provides a framework for subsequent theory testing and building only, and prior to that refinement process has no status beyond providing a 
language in terms of which one can begin to probe and express a growing understanding of the existing literature and observational studies.

\section{Establishment of Cognitive Elements}

Using the approach of Smith et al. (1994), an initial list of the object-related cognitive elements (noun, noun phrases and adjectival noun phrases) and action-related cognitive elements (verbs and verb plus adverbial phrases) used in each cognitive conceptual model was created. This initial list was then rationalised, with an aim of removing duplicates and synonyms. The list of nouns and verbs remaining constituted the set of cognitive elements (terms to which meaning must have been ascribed by any actors undertaking the activities in the cognitive conceptual model for these activities to take place). A list of the major cognitive elements for the activities appearing in Figures 3 to 7 , so developed is presented in Table 1, and has been used, as discussed below, as a means of comparing the model with our observational studies of problem solving behaviour.

Cognitive elements lessen the burden of conducting the comparison with protocol data by lending themselves easily to identification of areas of the protocol transcription which are potentially associated with model activities, by identification of utterances which reflect that the designers are attempting to deal with concepts which could be mapped to cognitive elements.

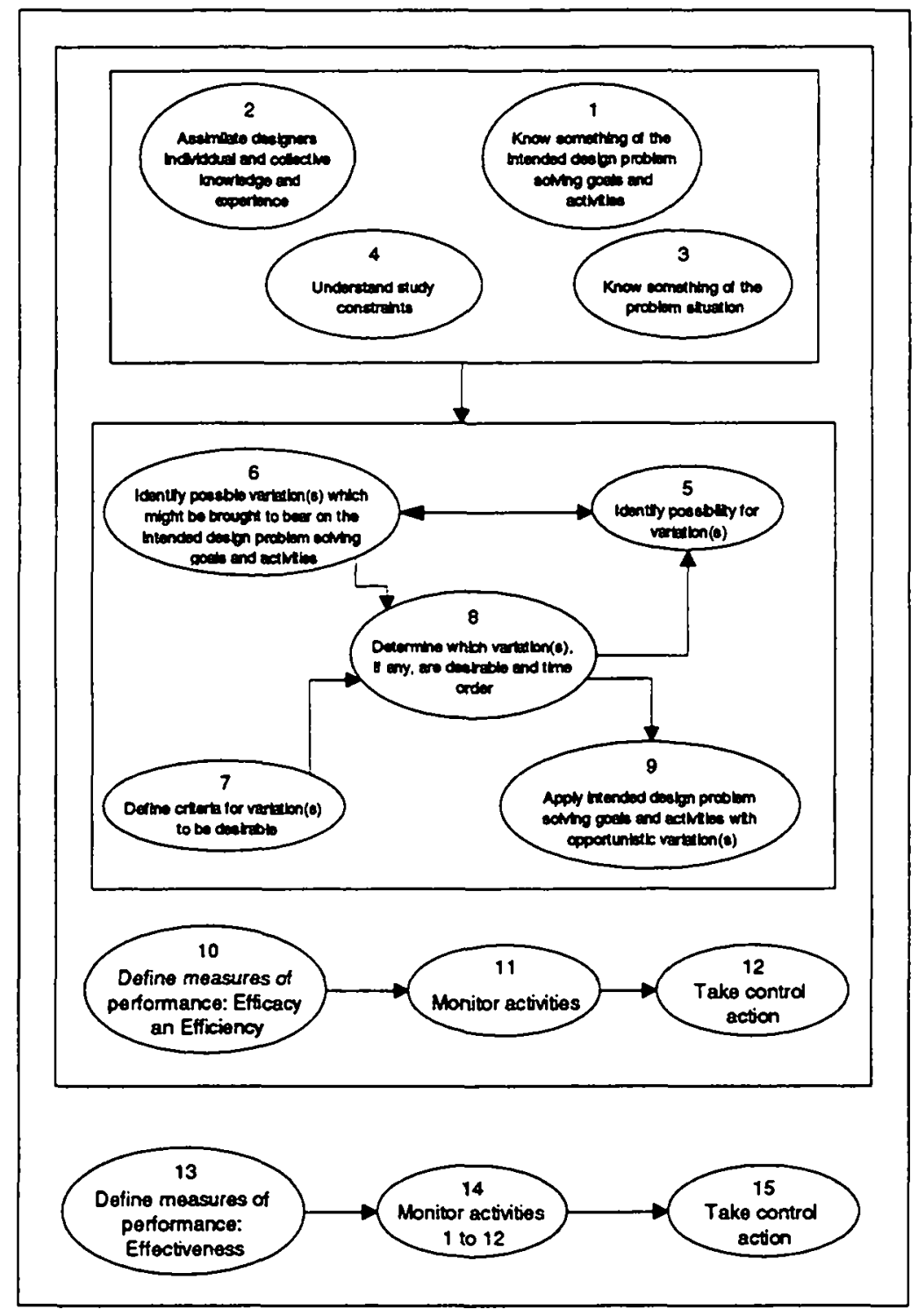

Figure 2. A system to make opportunistic variations to intended design problem solving goals and activities 


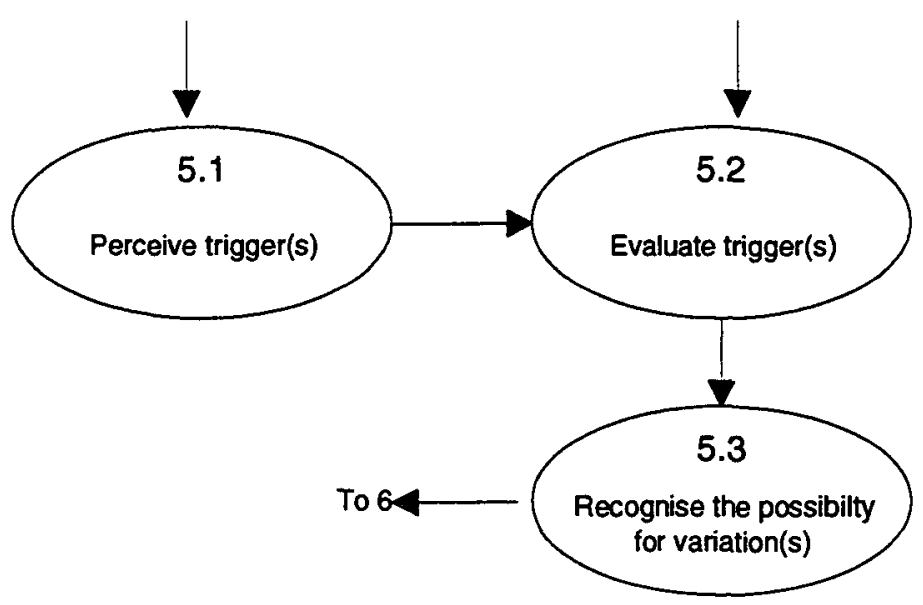

Figure 3. Activity 5 - Identify possibility for variation(s)

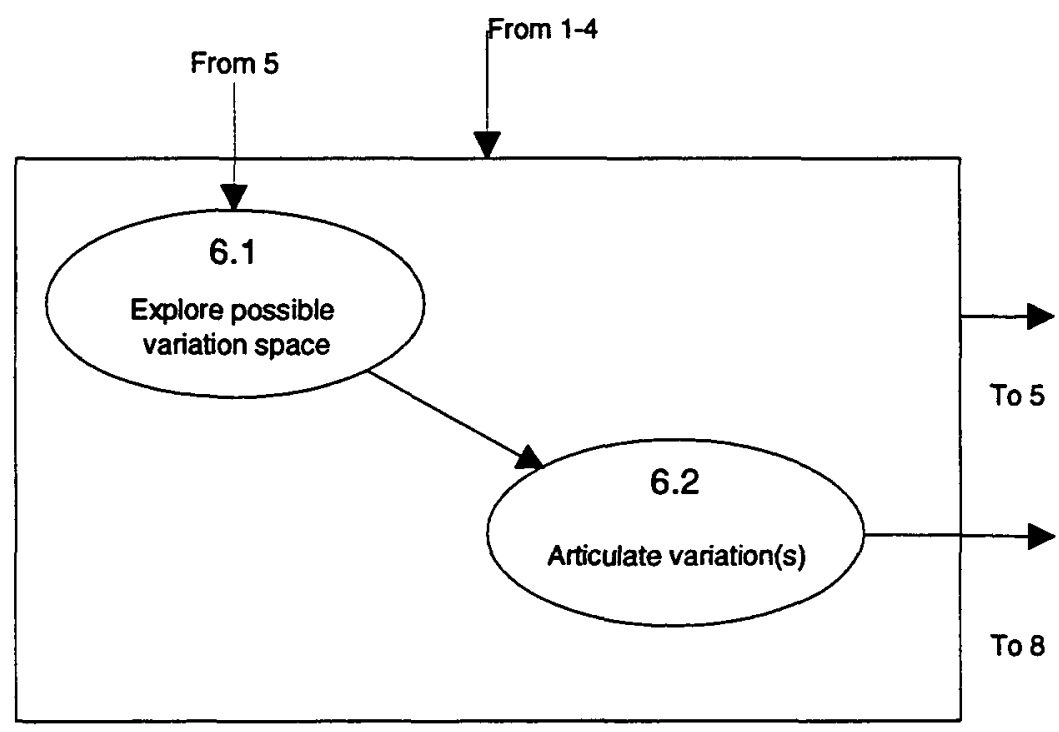

Figure 4. Activity 6 - Identify possible variation(s) which might be brought to bear on the intended design problem solving goals and activities 


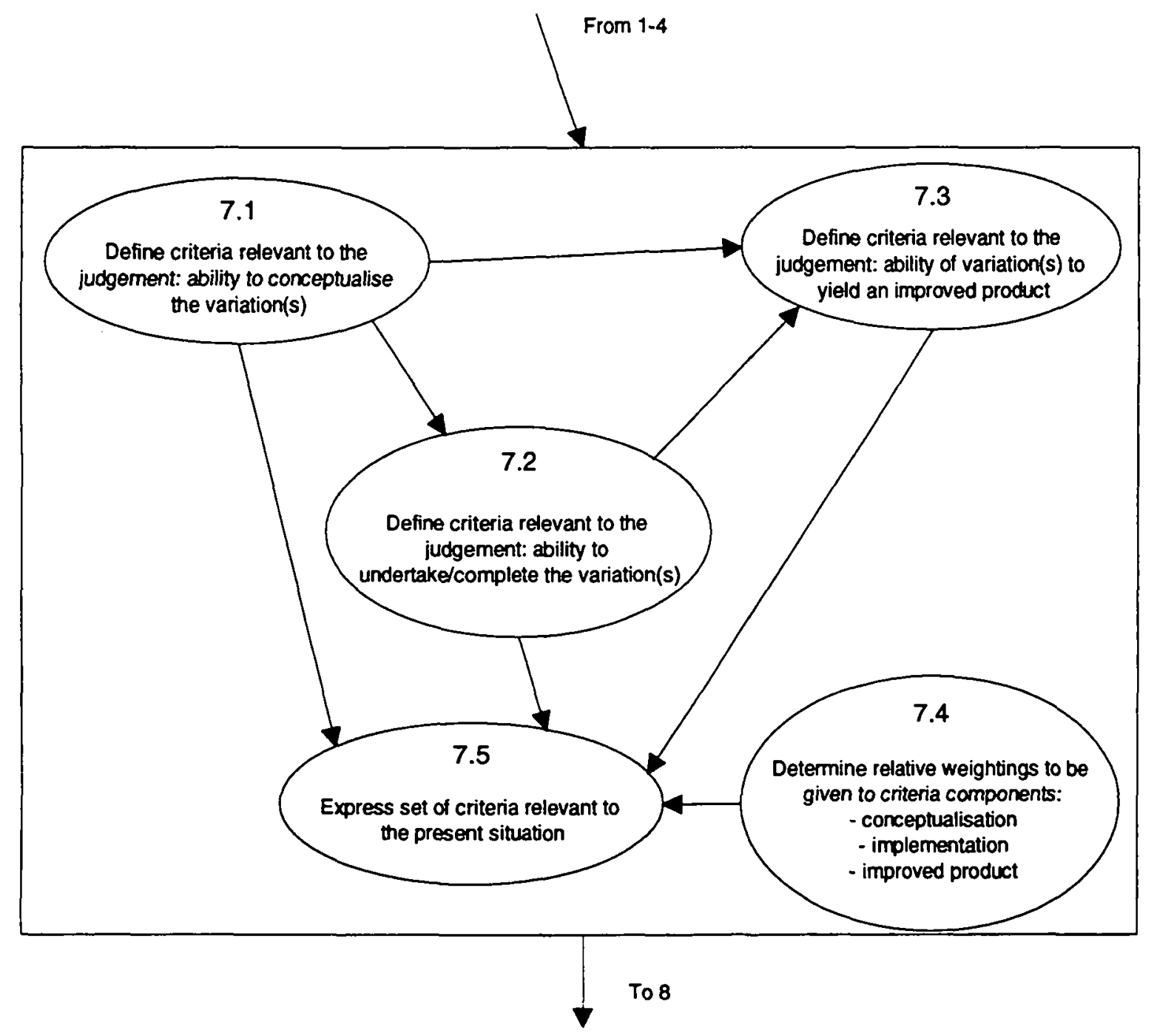

Figure 5. Activity 7 - Define criteria for variation(s) to be desirable

TOWARDS REFINEMENT OF THE MODEL OF OPPORTUNISM

As discussed above, the initial model reported must now be subjected to a process of iterative comparison with the existing literature and with empirical data, and must be modified to better reflect opportunistic behaviour in the real world. It is beyond the capacity of the present paper to describe the totality of this work. To indicate the nature of research in progress however, we present below an application of the model to the interpretation of a sample verbal protocol.

An Empirical Study using Simultaneous Verbalisation

During a period of seven weeks, comprehensive observation of a three member team involved in developing a strategic information system plan for the Office of a University Department Head was undertaken, with a view to providing a basis for improved support of office functions. The stated intended activity sequence of the design team was to pursue the preparation of a strategic information system plan in accord with the seven stage process of SSM (Checkland, 1981), adopting a comparison phase which utilised the Maltese Cross technique of Wilson (1990). The three designers were asked to verbalise their thoughts during their design problem solving activity, without intervention (ie., concurrent verbal protocol as per Ericsson \& Simon, 1980; also used by Guindon et al., 1987; Guindon \& Curtis, 1988; Visser, 1987, 1988, 1990b, 1990c, 1991a, 1991c, 1992a). 


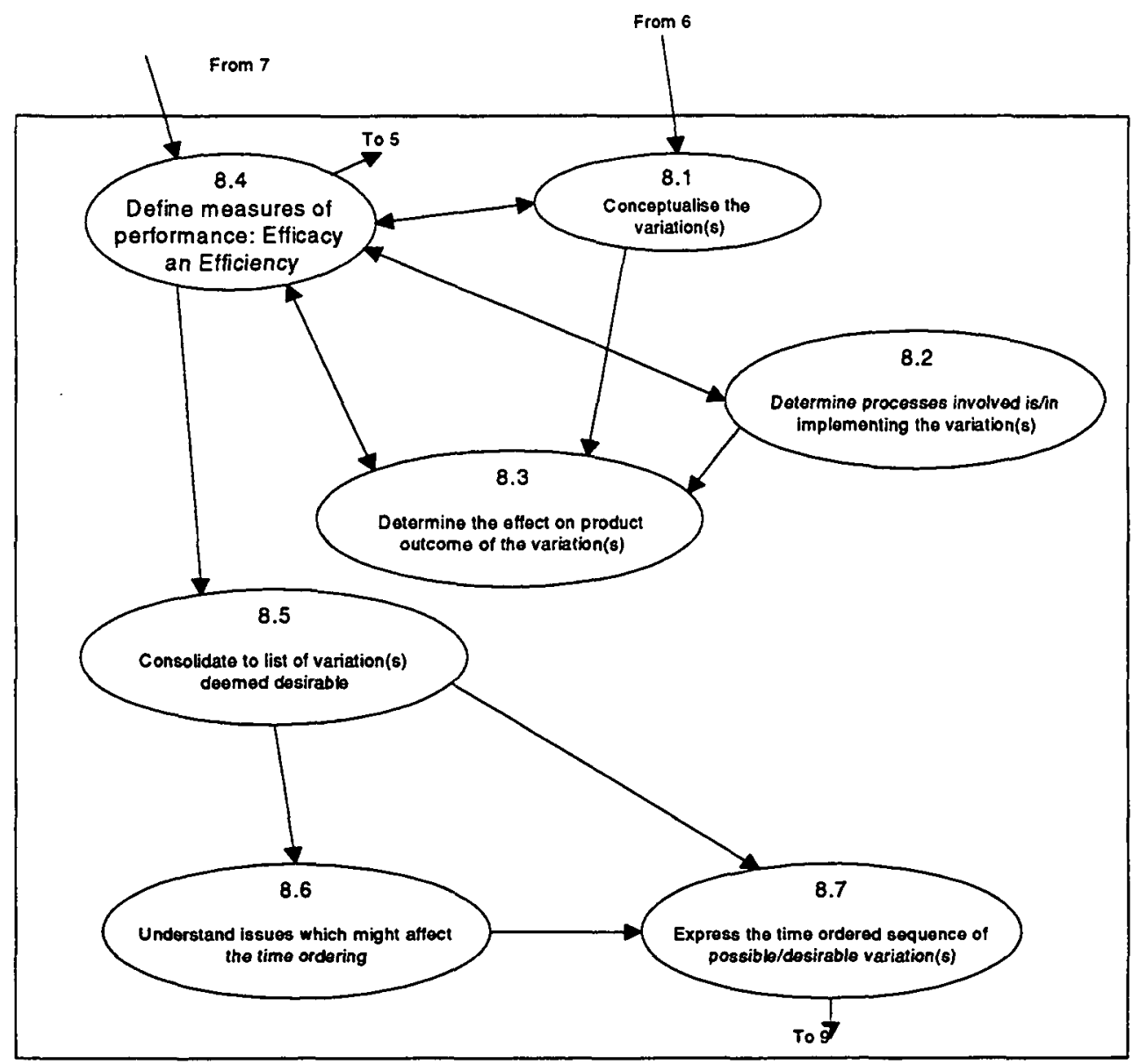

Figure 6. Activity 8 - Determine which variation(s), if any, are desirable and time order

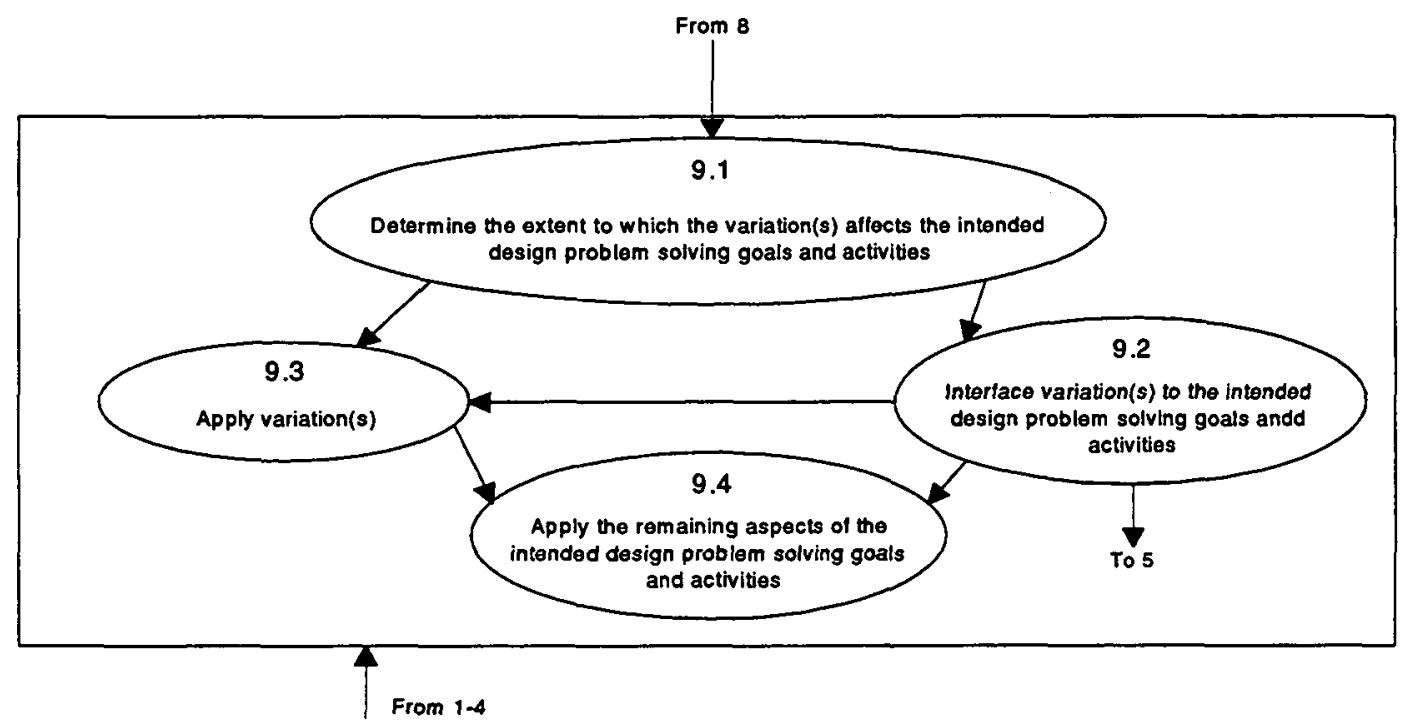

Figure 7. Activity 9 - Apply intended design problem solving goals and activities with opportunistic variation(s) 
Table 1. Cognitive elements (a system to make opportunistic variations to intended design problem solving goals and activities)

\begin{tabular}{|l|l|}
\hline Activity No & Elements \\
\hline 5.1 & A perceived trigger \\
\hline 5.2 & An evaluation of a trigger \\
\hline 5.3 & An acknowledgment of the possibility for variation \\
\hline 6.1 & An appreciation of the scope of possible variation(s) \\
\hline 6.2 & List of possible variations \\
\hline 7.1 & Set of criteria for assessing the extent to which a variation can be conceptualised \\
\hline 7.2 & Set of criteria for assessing the extent to which a variation can be implemented \\
\hline 7.3 & $\begin{array}{l}\text { Set of criteria for assessing the extent to which a variation can yield an improved } \\
\text { product }\end{array}$ \\
\hline 7.4 & $\begin{array}{l}\text { A position on the relative importance of conceptualisation, implementation and product } \\
\text { improvement in deciding whether to proceed with a variation }\end{array}$ \\
\hline 7.5 & List of criteria relevant to assessing the desirability of variations in the present situation \\
\hline 8.1 & A conceptualisation of the nature of a variation \\
\hline 8.2 & List of processes involved in implementing a variation \\
\hline 8.3 & List of effects on product outcome of a variation \\
\hline 8.4 & A determination, for each possible variation, of its desirability \\
\hline 8.5 & List of variations deemed desirable \\
\hline 8.6 & An understanding of issues which might affect the time ordering of variations \\
\hline 8.7 & Time-ordered list of variations deemed desirable \\
\hline 9.1 & $\begin{array}{l}\text { An understanding of the extent to which variations affect the intended design problem } \\
\text { solving goals and activities }\end{array}$ \\
\hline 9.2 & An interface of variations to the intended design problem solving goals and activities \\
\hline 9.3 & Variations which have been implemented \\
\hline 9.4 & $\begin{array}{l}\text { A resumption of the remaining aspects of the intended design problem solving goals and } \\
\text { activities }\end{array}$ \\
\hline & \\
\hline
\end{tabular}

\section{The Designers}

The three designers were enrolled in a Master's level course in information technology. They were rated highly competent, as assessed by academic record prior to joining the course, and subject performance throughout the course. Designer D1 had more than ten years professional experience in information systems analysis along with a degree in electrical engineering. Designer D2 had worked as a lecturer in information

a. systems with the University for more than ten years. Designer D3 had nearly ten years expertise in software development with a government organisation.

\section{Data Collection}

Concurrent verbal protocols were collected by videotaping designers during their design problem solving activities. All documents (such as interview notes with clients) and diagrams (such as rich picture and conceptual models) produced by designers were collected, and incorporated into the transcribed verbal protocols.

\section{Procedure - Protocol Analysis}

The procedure adopted to analyse the recorded protocols was as follows:

a. the intended design problem solving goals and activities of the team (strategic information systems planning according to the seven stage process of SSM) were matched to utterances in the transcription;

b. potential shifts in design problem solving goals and activities were highlighted by identification of utterances in the transcribed verbal protocols which reflected that the team was investigating concepts which could be mapped to the cognitive elements listed in Table 1 ; 
c. the transcription in the vicinity of each potential shift so identified was examined in detail, in the light of the intentions determined at step (a) above, to identify whether it constituted a variation from previously intended design problem solving goals and activities;

d. the sources giving rise to identified variations, and the subsequent activities as they evolved, were matched to activities 5 to 9 of Figures 3 to 7 ; and

e. activities not compatible with Figures 3 to 7 were noted for subsequent consideration.

\section{Data Analysis - Validation}

One excerpt from the empirical study is presented in Appendix B, and its interpretation is discussed below, to give some indication of both the utility of the model, and the iterative refinement process which has commenced.

Table 2 presents a comparison of the protocol (from Appendix B) with the model in a simple tabular form, analogous to the 'formal questioning' technique of Checkland \& Scholes (1990). As shown, a table of model defined questions was used, probing the extent to which the activities in the model (ie., $5.1,5.2,5.3, \ldots 9.4$ ) were carried out in the situation under study (ie., in this case the protocol excerpt illustrated in Appendix B). For example, activity 5.1 ("perceive trigger(s)"), with associated cognitive element "a perceived trigger", can be mapped in the protocol excerpt (Appendix B) to designer $\mathrm{D} 1$ noticing service provision problems being faced by part time evening students. Activity 5.2 ("evaluate trigger(s)"), with associated cognitive element "an evaluation of a trigger", can be mapped to designer D1's elaborate description of elements of the noticed problematic service. Similarly, activities 5.3 to 9.4 were mapped, giving rise to the interpretation illustrated in Table 2 . More recent work (Smith et al., 1994) discusses alternate graphical techniques for displaying this comparison, which are closer to Checkland's notion of 'model overlay' (Checkland \& Scholes, 1990).

\section{Discussion}

The excerpt analysed in Table 2 is but one, selected as representative of the extensive analysis of collected protocols which is in progress. Some tentative conclusions, drawn from analysis to date, include:

- There is no explicit evidence of designers exploring more than one variation option at any instant. The status of activity 6.1 , and the subsequent activities involved with sequencing variations (activities $8.5,8.6$ and 8.7 ) is therefore in question. Designers in the studies analysed to date have responded to a triggering event by exploring a single possible variation in depth, to the point where it has been either accepted and actioned, or rejected. Evidence from the design literature tends to suggest that such a depth-first strategy is characteristic of novice designers, whilst experts tend to adopt a breadth-first strategy. The studies we have analysed have involved designers of substantial experience in information systems analysis and design, although they have had only limited experience in the chosen problem solving methodology (SSM). Further studies, involving more experienced SSM practitioners, are planned to establish whether the above behaviour is generalisable or is simply an artefact of the limited specific methodological experience base of the subjects;

- There is only weak evidence of designers determining explicitly their criteria for assessing whether a variation of goal or activity will be desirable, and there is no explicit evidence that designers weight the various criteria, prior to commencing the variation (activities 7.1-5). More often such criteria and weightings as we have been able to recognise have been identified by reference to the talk-aloud protocol collected as designers implement their proposed variation. This finding is consistent with the studies of Hayes-Roth (1980), which concluded that criteria used by designers when taking decisions are often not stated explicitly, but instead are implicit, and become apparent only as designers undertake activities which follow from the taking of those decisions. One possible implication of such a finding would be that environments which support the up-stream phases of design might encourage the explicit expression of criteria, and weights given by designers to those criteria, prior to the taking of decisions to vary design goals and/or activities; 
- There is little evidence of designers considering explicitly the extent to which a possible variation will affect their previously intended goals and activities (activities 9.1-2). Such 'interfacing' of variations to the previous intentions of the designers seems to be handled implicitly within the subsequent processes of applying the variation and then resuming problem solving according to previously intended goals and activities. If confirmed, this finding has implications for the provision of tool support to the upstream phases of design. Specifically, one could envision support which enables designers to explore the effects of deviating from a current intended path (eg, by simulating the impact of a variation scenario).

Consolidation of these conclusions, and the modification of the model presented in Figures 2 to 7 which must consequently be made, awaits completion of a program of extensive literature comparison and empirical study which is in progress.

Table 2. Model based analysis of opportunistic behaviour (see excerpt in Appendix B)

\begin{tabular}{|c|c|}
\hline Activity & Observation \\
\hline $\begin{array}{l}\text { 5.1 - Perceive } \\
\text { trigger(s) }\end{array}$ & D1 noticed circumstances surrounding service provision to part time evening students. \\
\hline $\begin{array}{l}5.2 \text { - Evaluate } \\
\text { trigger(s) }\end{array}$ & $\begin{array}{l}\text { D1 described the noticed problem in detail, elaborating elements of the problematic } \\
\text { service to part time students. }\end{array}$ \\
\hline $\begin{array}{l}5.3 \text { - Recognise the } \\
\text { possibility for } \\
\text { variation(s) }\end{array}$ & $\begin{array}{l}\text { D1, based on the above evaluation, recognised the possibility to incorporate handling of } \\
\text { the noticed problem as a recommendation in the study. Implicit in the subsequent } \\
\text { dialogue is the potential for the team to attempt to formulate a recommendation now (an } \\
\text { ad hoc recommendation), rather than allow recommendations to emerge as a matter of } \\
\text { course later in the study as an outcome of SSM based analysis. }\end{array}$ \\
\hline $\begin{array}{l}6.1-\text { Explore pos } \\
\text { variation space }\end{array}$ & Only one possible variation (ie., formulate an ad hoc recommendation) was explored. \\
\hline $\begin{array}{l}6.2 \text { - Articulate } \\
\text { variation(s) }\end{array}$ & $\begin{array}{l}\text { The possibility of formulating an ad boc recommendation was articulated only } \\
\text { implicitly, as D1 and D3 began together to explore a possible recommendation (that } \\
\text { each staff member of the Office should answer student queries on a rotational basis } \\
\text { throughout the week). }\end{array}$ \\
\hline $\begin{array}{l}7.1 \text { - Define criteria: } \\
\text { ability to conceptualise } \\
\text { the variation(s) }\end{array}$ & $\begin{array}{l}\text { This excerpt offers no support for this activity. The designers had no apparent problem } \\
\text { in appreciating the notion of formulating an ad hoc recommendation and so the issue of } \\
\text { criteria did not arise. We believe that there will be evidence of this activity only when } \\
\text { designers are faced with a large cognitively taxing variation. }\end{array}$ \\
\hline $\begin{array}{l}7.2 \text { - Define criteria: } \\
\text { ability to undertake and } \\
\text { complete the } \\
\text { variation(s) }\end{array}$ & $\begin{array}{l}\text { This excerpt offers no support for this activity. The designers had no apparent problem } \\
\text { in understanding the mechanism of formulating an ad hoc recommendation and so the } \\
\text { issue of criteria did not arise. We believe that there will be evidence of this activity only } \\
\text { when designers are faced with a large cognitively taxing variation. }\end{array}$ \\
\hline $\begin{array}{l}7.3 \text { - Define criteria: } \\
\text { ability of variation(s) } \\
\text { to yield an improved } \\
\text { product }\end{array}$ & $\begin{array}{l}\text { Explicitly stated in the last sentence of the excerpt are criteria related to product, } \\
\text { against which the quality of any recommendation, ad hoc or as a result of detailed } \\
\text { analysis, will be assessed (ie., a recommendation must lead to resolution of student } \\
\text { queries and productivity improvement). In addition, D3 found the notion of answering } \\
\text { queries on a rotational basis to be constrained by the different job functions of each staff } \\
\text { member (eg, the secretary could not be expected to answer all queries relating to the } \\
\text { Computer Science Department as well as Information Technology). Designer D3 had } \\
\text { indirectly defined a further product-based criteria (ie., acceptable routing of student } \\
\text { queries). }\end{array}$ \\
\hline $\begin{array}{l}7.4 \text { - Determine } \\
\text { relative weightings }\end{array}$ & $\begin{array}{l}\text { There is no evidence of the designers explicitly or implicitly weighting any of the above } \\
\text { various product based criteria more highly than others. }\end{array}$ \\
\hline $\begin{array}{l}7.5 \text { - Express set of } \\
\text { criteria relevant to the } \\
\text { present situation }\end{array}$ & $\begin{array}{l}\text { There is no explicit statement of criteria prior to discussing possible ad hoc } \\
\text { recommendations, however the product-based criteria discussed at activity } 7 \text { above were } \\
\text { cited by the designers as they attempted to formulate a recommendation. }\end{array}$ \\
\hline $\begin{array}{l}8.1 \text { - Conceptualise the } \\
\text { variation(s) }\end{array}$ & $\begin{array}{l}\text { This excerpt offers no support for this activity. The designers had no apparent problem } \\
\text { in appreciating the notion of formulating an ad hoc recommendation. }\end{array}$ \\
\hline
\end{tabular}




\begin{tabular}{|c|c|}
\hline $\begin{array}{l}8.2 \text { - Determine } \\
\text { processes involved in } \\
\text { implementing the } \\
\text { variation(s) }\end{array}$ & $\begin{array}{l}\text { The designers entered debate on a possible ad hoc variation with no apparent } \\
\text { consideration of how their discussions might proceed. }\end{array}$ \\
\hline $\begin{array}{l}8.3 \text { - Determine the } \\
\text { effect on product } \\
\text { outcome of the } \\
\text { variation(s) }\end{array}$ & $\begin{array}{l}\text { It was determined that implementing the ad hoc recommendation which was emerging } \\
\text { would lead to productivity improvement and resolution of student queries, as evident } \\
\text { from the last sentence in the excerpt. The productivity improvement which designer D3 } \\
\text { referred to, arises from allocating a day to each staff member on a rotational basis, } \\
\text { which effectively ensures that on a day when it is the secretary's tum to answer queries, } \\
\text { the other two staff members are going to have an uninterrupted working day. The } \\
\text { resolution of student queries can be achieved by altering the roster time of staff } \\
\text { members, for example, asking the staff member who's turn it is to answer student } \\
\text { queries to start at } 9: 00 \text { and finish at } 6: 00 \text { or start at } 10: 00 \text { and finish at } 6: 00 \text {, as } \\
\text { mentioned in the excerpt. }\end{array}$ \\
\hline $\begin{array}{l}8.4 \text { - Apply criteria to } \\
\text { determine whether the } \\
\text { variation(s) is/are } \\
\text { desirable }\end{array}$ & $\begin{array}{l}\text { Having applied the product-based criteria, the designers accepted that formulating an ad } \\
\text { hoc recommendation and writing it directly into the study outcomes was desirable. }\end{array}$ \\
\hline $\begin{array}{l}8.5 \text { - Consolidate to a } \\
\text { list of variation(s) } \\
\text { deemed desirable }\end{array}$ & Only one variation (ad hoc recommendation formulation) was considered. \\
\hline $\begin{array}{l}8.6 \text { - Understand issues } \\
\text { which might affect the } \\
\text { time ordering }\end{array}$ & As there was only one variation, time ordering was not an issue. \\
\hline $\begin{array}{l}8.7 \text { - Express the time } \\
\text { ordered sequence of } \\
\text { possible/desirable } \\
\text { variation(s) }\end{array}$ & As there was only one variation, sequencing was not an issue. \\
\hline $\begin{array}{l}9.1 \text { - Determine the } \\
\text { extent to which the } \\
\text { variation(s) affects the } \\
\text { intended design } \\
\text { problem solving goals } \\
\text { and activities }\end{array}$ & $\begin{array}{l}\text { There was no explicit consideration of the effect that formulating an ad hoc } \\
\text { recommendation to cover this issue might have on the remainder of the study, although } \\
\text { implicitly it led to a re-scoping of work (see below). }\end{array}$ \\
\hline $\begin{array}{l}9.2 \text { - Interface } \\
\text { variation(s) to the } \\
\text { intended design } \\
\text { problem solving goals } \\
\text { and activities }\end{array}$ & $\begin{array}{l}\text { An implicit re-scoping of the study did take place, in that there was no further discussion } \\
\text { of the issue of handling part-time student enquires in the transcripts of the remaining } \\
\text { SSM study, effectively removing the development of SSM conceptual models etc. } \\
\text { relevant to this issue from the intended study activities. }\end{array}$ \\
\hline 9.3 - Apply variation(s) & $\begin{array}{l}\text { The designers proceeded to formulate an ad hoc recommendation, without reference to } \\
\text { the modelling stages of SSM. The final recommendation read: "Organise a roster for an } \\
\text { office member to start work at 10:00am and finish at } 6: 30 \mathrm{pm} \text { so evening students can } \\
\text { access the Office. Additionally, a system of routing the query to the right person must } \\
\text { be instituted". }\end{array}$ \\
\hline $\begin{array}{l}9.4 \text { - Apply the } \\
\text { remaining aspects of } \\
\text { the intended design } \\
\text { problem solving goals } \\
\text { and activities }\end{array}$ & $\begin{array}{l}\text { The designers resumed their rich picture building (Stage } 2 \text { of SSM), which they had } \\
\text { suspended while processing the above variation (ie., formulation of an ad hoc } \\
\text { recommendation to cover a specific issue). }\end{array}$ \\
\hline
\end{tabular}

\section{Research Reflections}

TheoryBuilder (Smith et al., 1994) has supported the process of cognitive model building, from initial model conception to model testing against empirical data. TheoryBuilder circumvented many of the problems germane to theory generation using orthodox psychological techniques, eg, verbal protocol analysis and experimental method, where problems arise in part due to a lack of support for data analysis and lack of a theory from which to generate testable hypotheses, respectively. 
In sympathy with TheoryBuilder's origins in SSM, the process of model development was discretionary, and hence the resultant model reflects only a partial interpretation of the data on opportunism.

With respect to the model development process, work is ongoing in exploring opportunism from more than one methodological perspective. TheoryBuilder is facilitating current research in exploring cognitive conceptual models through processes characterised by the development of multiple transformations, multiple root definitions and distinct cognitive conceptual models.

With respect to the model per se, work is ongoing in further decomposing model activities and validating the activities against suitable empirical data. Furthermore, some research is focusing on the generalisability of the model to other domains and design tasks and the implications, for design tools, of opportunistic behaviour.

\section{SUMMARY AND OUTLOOK}

In this paper, we have expressed the need to focus attention on the concept of opportunism, which is rapidly gaining recognition in a variety of independent problem domains. Numerous studies of opportunism have been reviewed and a tentative definition of the phenomenon has been provided.

Based upon that definition, and using the modelling principles which underpin the Lancaster Soft Systems Methodology, an initial model of opportunistic behaviour has been proposed. The model provides a framework for subsequent theory testing and building only, and prior to that refinement process has no status beyond providing a language in terms of which one can begin to probe and express a growing understanding of the existing literature and observational studies. The utility of some activities of the initial model has been demonstrated through its use to interpret an empirical study conducted by the authors. Results are very promising.

Further research in progress seeks to:

- refine the model by additional empirical study;

- establish the extent to which this model subsumes models developed by other authors; and

- focus on select model activities with a view to generating useful taxonomies of opportunistic behaviours, and investigating the consequences for support of up-stream design problem solving.

\section{ACKNOWLEDGMENT}

The first author would like to acknowledge support gained for the above research through an Australian Postgraduate Research Award. The second and third authors acknowledge support from the Australian Research Council.

\section{REFERENCES}

Adelson, B. and Soloway, E. (1985) "The role of domain experience in software design", IEEE Transactions on Software Engineering, Vol SE-11, No 11, pp 1351-1360.

Bimbaum, L. (1985) "A short note on opportunistic planning and memory in arguments", in Proceedings of the Ninth International Joint Conference on Artificial Intelligence, Vol 1, pp 281-283.

Bisseret, A., Figeac-Letang, C. and Falzon, P. (1988) "Modelling opportunistic reasonings: The cognitive activity of traffic signal setting technicians", Research Report No 898, Rocquencourt: INRIA.

Broadbent, D. (1993) "Planning and Opportunism", The Psychologist: Bulletin of the British Psychological Society, Issue 6, pp 54-60.

Carroll, J. M., Thomas, J. C. and Malhotra, A. (1979) "Clinical-experimental analysis of design problem solving", Design Studies, Vol 1, pp 84-92.

Checkland, P. (1981) Systems Thinking, Systems Practice, Wiley, Chichester.

Checkland, P. and Scholes, J. (1990) Soft Systems Methodology in Action, Wiley, Chichester.

Davies, L. J. (1989) "Designing from ill-defined problems", International Journal of Information Management, Vol 9, pp 199-208.

Davies, L. and Ledington, P. (1991) Information in Action, Soft Systems Methodology, Macmillan Information Systems Series. 
Davies, S. P. (1991) "Characterising the program design activity: Neither strictly top-down nor globally opportunistic", Behaviour and Information Technology, Vol 10, pp 173-190.

Ericsson, K. A. and Simon, H. A. (1980) "Verbal Reports as Data", Psychological Review, Issue 87, pp 215- 251.

Eunson, B. (1988) Behaving: Managing yourself and others, McGraw-Hill Book Company, Australia.

Guindon, R. (1990a) "Designing the design process: Exploiting opportunistic thoughts", HumanComputer Interaction, Vol 5, pp 305-344.

Guindon, R. (1990b) "Knowledge exploited by experts during software system design", International Journal of Man-Machine Studies, Vol 33, pp 279-304.

Guindon, R. and Curtis, B. (1988) "Control of cognitive processes during design: What tools would support software designers ?", in Proceedings of CHI'88, Washington, D.C., ACM Press, Addison-Wesley Publishing, pp 263-268.

Guindon, R., Krasner, H. and Curtis, B. (1987) "Breakdowns and Processes during the early activities of software design by professionals", in Olson, G. M., Sheppard, C. and Soloway, E. (eds.), Empirical Studies of Programmers: Second Workshop, Norwood, NJ: Ablex, pp 65-82.

Hayes-Roth, B. (1980) "Human planning processes", Technical Report No R-2670-ONR, Rand Corporation, Santa Monica, California.

Hayes-Roth, B. and Hayes-Roth, F. (1978) "Cognitive processes in planning", Technical Report No R-2366- ONR, Rand Corporation, Santa Monica, California.

Hayes-Roth, B. and Hayes-Roth, F. (1979) "A Cognitive Model of Planning", Cognitive Science, Vol 3, pp 275-310.

Huber, G. P. (1980) Managerial Decision Making, Glenview.

Jeffries, R., Turner, A.A., Polson, P. and Atwood, M. E. (1981) "The processes involved in designing software", inCog Anderson, J. R. (ed.), Cognitive Skills and their Acquisition, Hillsdale, NJ.: Erlbaum, pp 225-283.

Kant, E. (1985) "Understanding and automating algorithm design", IEEE Transactions on Software Engineering, SE-11, pp 1361-1374.

Kant, E. and Newell, A. (1984) "Problem solving techniques for the design of algorithms", Information Processing and Management, Vol 20, No 1-2, pp 91-118.

Lawson, B. (1980) How Designers Think, The Architectural Press Ltd: London, Eastview Editions, Inc: Westfield, New Jersey.

Malhotra, A., Thomas, J. C., Carroll, J. M. and Miller, L. A. (1980) "Cognitive processes in design", International Journal of Man-Machine studies, Vol 12, pp 119-140.

Nii, H. P. (1986) "Blackboard Systems: Blackboard Application systems, blackboard systems from a knowledge engineering perspective", The AI Magazine, Part 1, Summer 1986, pp 38-53, and Part 2, August 1986, pp 82-106.

Norcio, A. F. (1982) "Indentation, Documentation and Programmer Comprehension", in Proceedings of the Conference on Human Factors in Computer Systems, Gaithersburg, MD.

Parnas, D. L. and Clements, P. C. (1986) "A rational design process: How and why to fake it", IEEE Transactions on Software Engineering, Vol 12, pp 251-257.

Shneiderman, B. (1986) "Empirical Studies of Programmers: The Territory, Paths and Destinations", in Empirical Studies of Programmers, Ablex.

Simon, H. A. (1973) "The structure of ill-structured problems", Artificial Intelligence, Vol 4, pp 181-201

Smith, R., Howard, S., Sutherland, T., and Khushalani, A. (1994), "TheoryBuilder. A Behavioural Perspective on Modelling and Improving Systems Development", in Proceedings of the 1st Australian Seminar on Modelling and Improving System Development (OzMisD'94), Melbourne Australia, Feb 3-4 1994.

Sumiga, J. H. (1992) "Programming and Design", Technical Report No SIT-CS-1/92, Swinburne University of Technology, Melboume, Australia.

Ullman, D. G., Dietterich, T. G. and Stauffer, L. A. (1988) "A model of the mechanical design process based on empirical data", AI EDAM, Vol 2, pp 23-52.

Visser, W. (1987) "Strategies in programming programmable controllers: A field study on a professional programmer", in Olson, G., Sheppard, S. and Soloway, E. (eds.), Empirical studies of programmers: Second Workshop, Norwood, NJ: Ablex, pp 217-230. 
Visser, W. (1988) "Towards modelling the activity of design: An observational study on a specification stage", in Proceedings of the IF AC/IFIP/IEA/IFORS Conference on ManMachine systems, analysis, design and evaluation, Oulu, Finland, pp 249-254.

Visser, W. and Hoc, J. M. (1990a) "Expert software design strategies", in Hoc, J. M., Green, T., Samurcay, R. and Gilmore, D. (eds.), Psychology of Programming, London: Academic Press, pp 235-249.

Visser, W. (1990b) "Evocation and elaboration of solutions: Different types of problem-solving actions. An empirical study on the design of an aerospace artefact", in Kohonen, $T$. and Fogelman-Soulie, F. (eds.), COGNITIVA 90, At the crossroads of Artificial Intelligence, Cognitive Science and Neuroscience, Proceedings of the third COGNITIVA symposium, Amsterdam: Elsevier, pp 689-696.

Visser, W. (1990c) "More or less following a plan during design: Opportunistic deviations in specification", International Journal of Man-Machine studies, Special Issue: What programmers know, Vol 33, pp 247-278.

Visser, W. (1991a) "Designers' activities examined at three levels: Organisation, Strategies and Problem-solving processes", Knowledge Based Systems, Vol 5, No 1, pp 92-104.

Visser, W. (1991b) "Design activities through the research in a cognitive psychology team", Paper presented at the EdCAAD-workshop, Edinburgh.

Visser, W. (1991c) "The cognitive psychology viewpoint on design: Examples from empirical studies", in Gero, J. (ed.), Artificial Intelligence in Design, Oxford: Butterworth-Heinemann, pp 505524.

Visser, W. (1992a) "Planning and Organisation in expert design activities", (Paper submitted for publication in Jan 1992).

Visser, W. (1992b) "Design Organisation: There is more to expert knowledge than is dreamed of in the planner's philosophy", Research Report No 1765, Rocquencourt: INRIA.

Wilson, B. (1990) Systems: Concepts, methodologies and applications, Wiley, Chichester.

\section{APPENDIX A: Model Based Analysis of Literature Excerpt}

The following scenario has been proposed by Bimbaum (1985). It is interpreted in Table Al in terms of the activities included in the model displayed as Figures 3 to 7.

"Suppose a planner decides to go to the store to buy milk. In the course of executing that plan, while at the store, he notices that eggs are on sale. Realising that he will need eggs in the near future, he checks to see whether he has sufficient funds, and if he does, he buys some."

Table A1. Interpretation of Literature Excerpt

\begin{tabular}{|l|l|}
\hline Activity & Interpretation \\
\hline $\begin{array}{l}5.1 \text { - Perceive } \\
\text { trigger(s) }\end{array}$ & The planner notices eggs are on sale. \\
\hline $\begin{array}{l}5.2 \text { - Evaluate } \\
\text { trigger(s) }\end{array}$ & $\begin{array}{l}\text { The planner responds to having noticed eggs on sale, by realising his need for eggs in } \\
\text { the near future. }\end{array}$ \\
\hline $\begin{array}{l}5.3 \text { - Recognise the } \\
\text { possibility for } \\
\text { variation(s) }\end{array}$ & $\begin{array}{l}\text { The planner, based on the above evaluation, recognises the possibility to incorporate the } \\
\text { purchasing of eggs within his intended plan (which originally was to purchase milk } \\
\text { only). }\end{array}$ \\
\hline $\begin{array}{l}6.1 \text { - Explore possible } \\
\text { variation space }\end{array}$ & $\begin{array}{l}\text { Only one possible variation (ie., purchase eggs now) is explored. One could speculate } \\
\text { that in an expanded scenario, he might explore a number of possibilities (eg, buy eggs at } \\
\text { another shop, borrow some from a neighbour on the way home). }\end{array}$ \\
\hline $\begin{array}{l}6.2 \text { - Articulate } \\
\text { variation(s) }\end{array}$ & The possible variation in articulated form is "purchase eggs now". \\
\hline $\begin{array}{l}7.1-\text { Define criteria: } \\
\text { ability to conceptualise } \\
\text { the variation(s) }\end{array}$ & $\begin{array}{l}\text { The purchasing of eggs is an easy variation to conceptualise and so the issue of criteria } \\
\text { does not arise. }\end{array}$ \\
\hline $\begin{array}{l}7.2 \text { - Define criteria: } \\
\text { ability to undertake and } \\
\text { complete the } \\
\text { variation(s) }\end{array}$ & $\begin{array}{l}\text { The ability to undertake the variation (ie., purchasing eggs now), in the excerpt above is } \\
\text { to be judged according to the criterion that the purchase of milk must not be } \\
\text { compromised (by the purchase of eggs exhausting his funds). }\end{array}$ \\
\hline
\end{tabular}




\begin{tabular}{|c|c|}
\hline $\begin{array}{l}7.3 \text { - Define criteria: } \\
\text { ability of variation(s) } \\
\text { to yield an improved } \\
\text { product }\end{array}$ & $\begin{array}{l}\text { Implicit in the excerpt is the product-based criteria that there should be financial savings } \\
\text { (ie., eggs are on sale). It could also be speculated that since the planner has realised the } \\
\text { need for eggs in the near future, another product-based criteria might be time saving (ie., } \\
\text { benefit would result from avoiding a trip to the store in the near future to purchase } \\
\text { eggs). }\end{array}$ \\
\hline $\begin{array}{l}7.4 \text { - Determine } \\
\text { relative weightings }\end{array}$ & $\begin{array}{l}\text { Although not explicitly acknowledged, the scenario seems to suggest that the issue of } \\
\text { financial savings is strongly weighted in the decision to proceed. }\end{array}$ \\
\hline $\begin{array}{l}7.5 \text { - Express set of } \\
\text { criteria relevant to the } \\
\text { present situation }\end{array}$ & There is no explicit statement of criteria. \\
\hline $\begin{array}{l}8.1 \text { - Conceptualise the } \\
\text { variation(s) }\end{array}$ & The planner has no apparent problem in appreciating the notion of purchasing eggs now. \\
\hline $\begin{array}{l}8.2 \text { - Determine } \\
\text { processes involved in } \\
\text { implementing the } \\
\text { variation(s) }\end{array}$ & $\begin{array}{l}\text { The planner determines that he must check available funds as a component of } \\
\text { purchasing eggs now. }\end{array}$ \\
\hline $\begin{array}{l}8.3 \text { - Determine the } \\
\text { effect on product } \\
\text { outcome of the } \\
\text { variation(s) }\end{array}$ & $\begin{array}{l}\text { The planner determines that purchasing eggs now would lead to financial savings (as } \\
\text { eggs are on sale). We could speculate that he also determines that he will save himself } \\
\text { an extra trip to the store to purchase eggs in the near future. }\end{array}$ \\
\hline $\begin{array}{l}8.4 \text { - Apply criteria to } \\
\text { determine whether the } \\
\text { variation(s) is/are } \\
\text { desirable }\end{array}$ & $\begin{array}{l}\text { The planner decides that purchasing eggs now is in accord with his criteria (financial } \\
\text { savings, and will still allow him to purchase milk), and determines to purchase eggs } \\
\text { now. }\end{array}$ \\
\hline $\begin{array}{l}8.5 \text { - Consolidate to a } \\
\text { list of variation(s) } \\
\text { deemed desirable }\end{array}$ & Only one variation (ie., purchase eggs now) is considered. \\
\hline $\begin{array}{l}8.6 \text { - Understand issues } \\
\text { which might affect the } \\
\text { time ordering }\end{array}$ & As there is only one variation, time ordering is not an issue. \\
\hline $\begin{array}{l}8.7 \text { - Express the time } \\
\text { ordered sequence of } \\
\text { possible/desirable } \\
\text { variation(s) }\end{array}$ & As there is only one variation, sequencing is not an issue. \\
\hline $\begin{array}{l}9.1 \text { - Determine the } \\
\text { extent to which the } \\
\text { variation(s) affects the } \\
\text { intended design } \\
\text { problem solving goals } \\
\text { and activities }\end{array}$ & $\begin{array}{l}\text { The planner determines that purchasing eggs now will not affect his capacity to also } \\
\text { purchase milk. }\end{array}$ \\
\hline $\begin{array}{l}9.2 \text { - Interface } \\
\text { variation(s) to the } \\
\text { intended design } \\
\text { problem solving goals } \\
\text { and activities }\end{array}$ & There are no adaptation issues evident in this excerpt. No interfacing is needed. \\
\hline 9.3 - Apply variation(s) & The planner purchases the eggs. \\
\hline $\begin{array}{l}9.4 \text { - Apply the } \\
\text { remaining aspects of } \\
\text { the intended design } \\
\text { problem solving goals } \\
\text { and activities }\end{array}$ & $\begin{array}{l}\text { The planner purchases the milk as per his intended plan and leaves the store with milk } \\
\text { and eggs. }\end{array}$ \\
\hline
\end{tabular}




\section{APPENDIX B: Protocol Excerpt}

D1 : Another thing that I noticed as the basis of our recommendations. Most part timers, evening students specially, come in at 4:30 at the earliest, often 5:00, and they cannot find anyone to relate to or hand in assignments, ask questions, do anything of that sort, especially in the early days when they don't know whether they are coming or going. They don't know who to speak to, where to get things organised and there is nobody to help them at all. So whilst they are the clients of the Office of the Computer Science Department they can never use it because whenever they are on the sceneit is closed.

D3 : Actually, one of our recommendations is interruptions; somebody answers student queries on Monday, somebody else on Tuesday, somebody else's turn on Wednesday. Now, I think one of the reasons that precludes that is everyone has a different set of job functions and if it is the secretary's turn to answer student queries for Information Technology students, she is not going to tap Information Technology.

D1 : Not the change. If she needs to do that, she may do it over the first couple of months until; look because queries are of two different nature, either they are of general nature or a specific nature. If they are of general nature, after a while, even if she is not able to answer them right away in the beginning, after a couple of months she will learn the responses. If they are of specific nature, then there are two ways of handling them. Either she liaises with whoever is responsible, acting as a buffer, which means sure enough his time will be taken. He will be interrupted but that is part of his job, but there will at least be a buffer there or if it is not of an urgent nature, she can tell the student or whoever to come back at a particular time when he is on the job. Either of the ways, I mean that's for them to decide. The recommendation however should be to introduce something like that because it will improve productivity tenfold, and the person who is on duty on a given day, rather than starting at 8:00, starts at 9:00 but finishes at 6:00, or starts at 10:00 and stays till 6:00. 